\title{
WIRELESS SENSOR NETWORKS FOR INTERNET OF THINGS BASED LABORATORY AUTOMATION SYSTEM
}

\author{
Vishnukumar V ${ }^{1}$, Satheesh Kumar M V', K N Madhusoodanan ${ }^{3}$ \\ ${ }^{I}$ Department of Instrumentation, Cochin University of Science and Technology, Cochin, India \\ ${ }^{2}$ Department of Computer Science, IHRD College, Payyapadi, India \\ ${ }^{3}$ Department of Instrumentation, Cochin University of Science and Technology, Cochin, India
}

\begin{abstract}
The model proposed in this paper is a system for sustaining a healthy atmosphere in experimental/research laboratories through the real time monitoring and controlling of environmental parameters with low power and simple hardware modules. Internet of things (IoT) is the key technology behind the system, creating an automated laboratory environment through wireless sensor networks. The system monitors a range of parameters such as room temperature, relative humidity, light intensity, smoke, gas leakage etc. with a minimum number of low power sensor nodes. And the system generates control signals for alerts and actuation controls depending upon the parameter levels. At the same time, all the laboratory parameter values are hosted to a web server for the real time dashboard monitoring and graphical representation, accessible from anywhere in the world. An important thing to be noted is that only open source platforms and languages are employed throughout the work such as Arduino IDE, PHP and MYSQL.
\end{abstract}

Keywords: Wireless Sensor Networks, Sensor Nodes, Internet of Things, Wi-Fi ****

\section{INTRODUCTION}

Currently, Internet of things (IoT) and automation concepts are gaining a lot of attention. It can be used to operate in a complex laboratory environment and can provide an intelligent way to improve work quality, productivity, human/machine healthcare, energy management etc.[1]. The proposed system in this work aims to monitor and control a set of parameters inside the selected laboratories in a research centre or institute. This advanced system makes the labs smart and intelligent, capable of making decisions adaptively according to the varying environmental parameters. The system uses multiple arrays of sensors called sensor nodes, capable of data acquisition, analysis, communication and actuation processes; hence collectively called a smart sensor.

In this work, we deploy multiple sensor nodes for chemical lab, LASER lab, and Distributed Control System (DCS) lab incorporating analog and digital calibrated sensors such as temperature/humidity sensor, light intensity sensor, smoke sensor, gas sensor etc., according to the different laboratory contexts. Every sensor node in each lab establishes a wireless channel with a locally placed $\mathrm{Wi}-\mathrm{Fi}$ router which acts as an access point of internet connectivity, constituting a star type wireless sensor network. The communication standard adopted in the sensor node is IEEE $802.11 \mathrm{~b} / \mathrm{g} / \mathrm{n}$, known as wireless fidelity (Wi-Fi), which uses $2.4 \mathrm{GHz}$ ISM band. We prefer Wi-Fi for local networks over zigbee(uses same frequency band) considering its broadcasting easiness and hardware simplicity. Although there were many microcontroller-Wi-Fi/Zigbee combined data hosting research works conducted previously [2][3][4][5], we developed a different system with a compact module for both data acquisition and communication. Here we set the sensor node as a client of the internet server "freelancewebdesigin.com"to which the multi sensor data are hosted. All these tasks up to the data hosting are done at the sensor node itself as per the embedded firmware loaded in the microcontroller. From there onwards, the data monitoring and graphical representation tasks are accomplished by the server end scripts [6].

\section{EXPERIMENTAL METHODS}

\subsection{System Architecture}

Developing an IoT based wireless sensor network system requires the integration and coordination of many hardware and software components. Fig. 1 shows the overall system architecture of the proposed wireless sensor network system that we have developed.

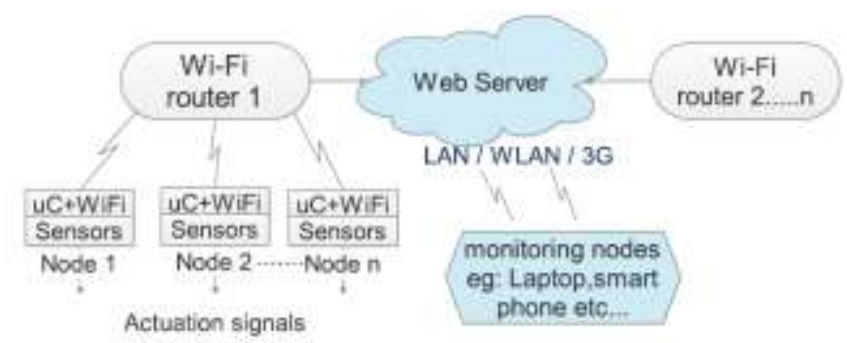

Fig.1. Overall System Architecture

Considering the factors such as technical features, power consumption, compatibility and economic benefits, we chose Wi-Fi network standard built upon $802.11 \mathrm{~b} / \mathrm{g} / \mathrm{n}$ for our work. The system consists of a number of distributed wireless sensor nodes, Wi-Fi routers and associated software 
platforms. We use a compact module ESP 8266 12E for data collection, analysis, control actuation and Wi-Fi network establishment. The integration of $\mathrm{Wi}-\mathrm{Fi}$ transceiver with microcontroller in a single package makes the system more power efficient, size compactness and cost saving. Each sensor node is incorporated with multiple sensors, a microcontroller and $\mathrm{Wi}-\mathrm{Fi}$ transceiver. The general block diagram of individual sensor nodes is shown in Fig.2.

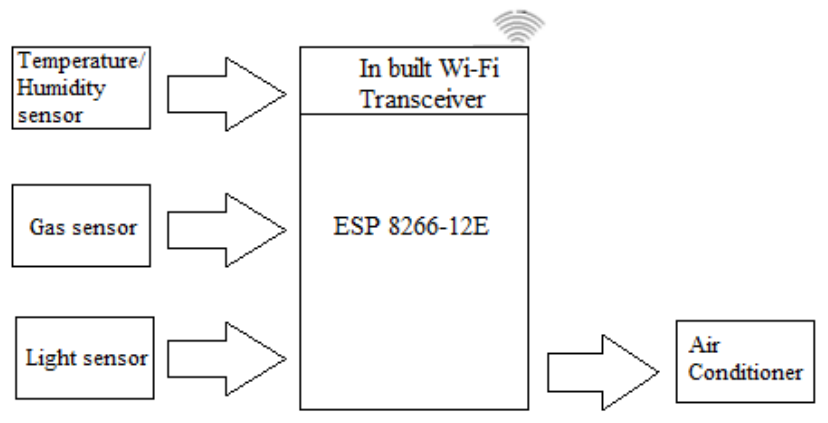

Fig.2. Sensor Node block diagram

ESP-12E Wi-Fi module is developed and industrialized by Ai-thinker Team. They integrated a 32 bit microprocessor Tensilica L106, which has higher performance and smaller size in comparison with ARM processors. It requires $3.3 \mathrm{Vdc}$ power supply and supports Clock speeds $80 \mathrm{MHz}$ and 160 $\mathrm{MHz}$. The module features on-chipTCP/IP protocol stack and RTOS compatibility for higher applications. On-board micro strip antenna integrated in the module enables $\mathrm{Wi}-\mathrm{Fi}$ communication with router within the network. Once established a Wi-Fi network connection, the channel acquires an IP address, which is accessible to its local network. The open source platform Arduino IDE is used for programming the ESP module. The embedded firmware in the controller of each sensor node handles sampling of sensor data in a certain well defined manner and communication with the server. For improving the feasibility of in-system firmware updates, a development board- Node MCU is employed. Node MCU is a USB powered board; on which ESP is mounted (SMT), provides the same pinout of ESP as shown in Fig.3.

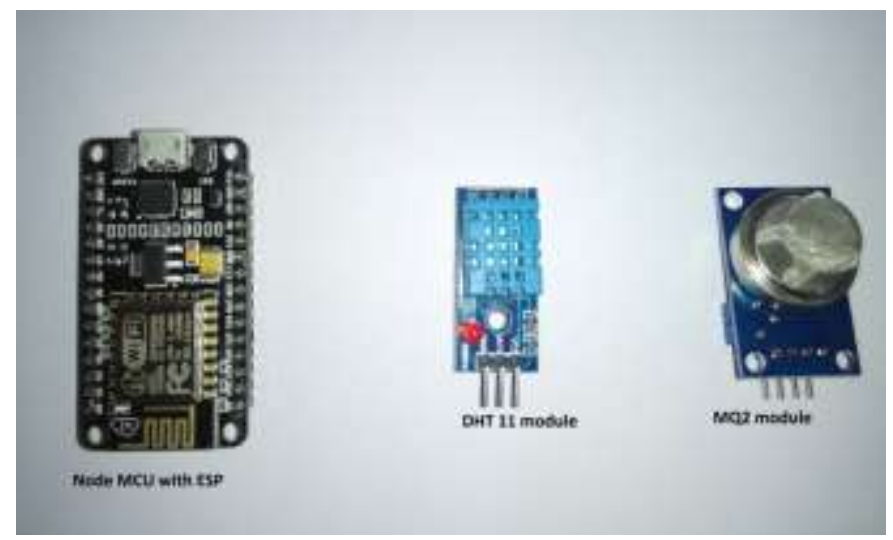

Fig.3. Wi-Fi and sensor modules

\subsection{System Implementation}

For the system demonstration, we deployed each sensor node at three laboratories, which requires different kinds of sensors according to the variety of parameters. In chemical lab, DHT11 (for room temperature and relative humidity) and MQ2 (for the concentration of LPG, Carbon monoxide $\&$ smoke) sensors are connected at the node. In DCS Instrumentation lab, only DHT 11 sensor is connected and there is a corrective mechanism to maintain the room temperature around $25-30^{\circ} \mathrm{C}$ (as per the DCS specification requirement) with air conditioner and relay mechanism. In LASER lab, DHT 11 and LDR (Light dependent resistor) sensors are placed since light and room temperature are the major concern of LASER lab and the dark room.

For the real time monitoring of varying parameters at the laboratory environment on a web page, we set up a web server with domain name "satheesh.freelancewebdesigin.com/epm". The page layouts and graphics of the web page is designed by using HTML (Hyper Text Mark up Language) and CSS (Cascade Style Sheet) scripts. A database is created in MySQL script for the continuous storage and update of the measurement data. The data handling tasks done at the server side are data feeding (to database), data manipulation and data representation. We built up separate PHP scripts for performing each task to improve the programming clarity while revising the scripts. Here we have two scripts named insert.php and view.php for data feeding and data representation respectively. We can term MySQL and PHP as as back end scripting languages. And HTML, CSS and JavaScript come under the category of front end user interface scripts.

\section{RESULTS AND DISCUSSIONS}

The entire web page includes data monitoring, graph plotting and data logging sections. The system is named as "SMART LAB", having sub windows- Home, View all data, View chart and View by node, as shown in Fig.4. Each sub window displays node-wise and parameter-wise data.

\subsection{Dashboard View and Data Logging}

As we have deployed three sensor nodes in this model, three dashboard panels are displayed on the monitoring window. In the data monitoring window, there are provisions for data downloading (as CSV file) and data clearance (up to date).

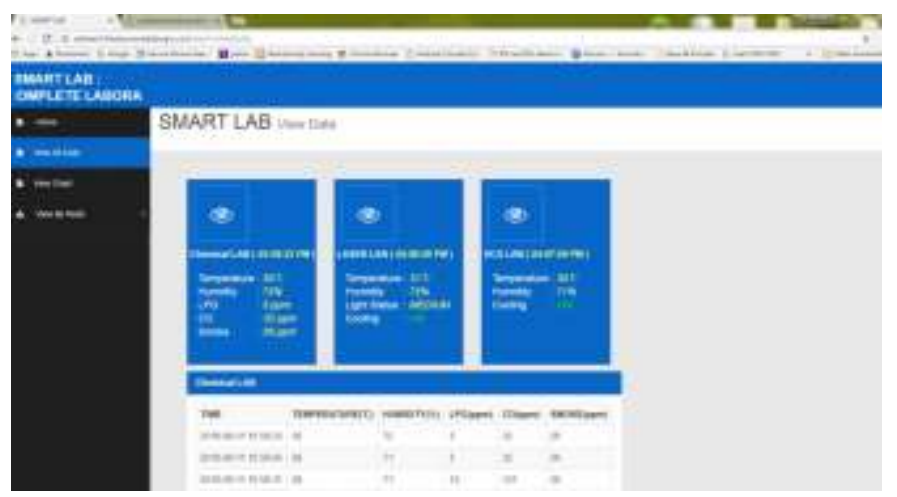

Fig.4. Dashboard display and parameter logging 
Also the display shows the cooling status of the air conditioner which is set to maintain the room temperature in the range of $25-30^{\circ} \mathrm{C}$. The refreshing interval provided for dashboard display is 4 seconds.

\subsection{Graph Analysis}

In the chart window, only temperature and humidity are plotted in terms of time, for which continuous graphical representation is more effective for analysis.

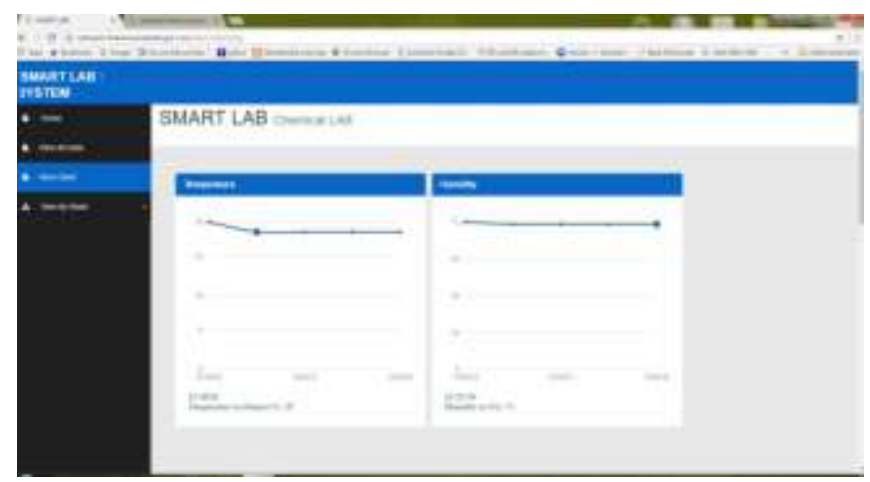

Fig.5. Temperature and Humidity plots

The refreshing interval provided for the chart is 20 seconds. Fig.5is the graphshowing temperature and humidity variation with time.

\section{CONCLUSION}

Over the last decade, wireless sensor networks and Internet of things became important to such an extent that a numerous real life applications have implemented recently. This paper could introduce a model of ultra-low power laboratory automation system that provides multiple parameter values with minimum number of sensors. The proposed architecture of the system explores the gathering of real-time measurements in accordance with the fluctuating laboratory environmental attributes and the system will be very useful for laboratory technicians. The system can be recommended to minimize the energy consumption of the WSN components. The research and development of the system has been accomplished and demonstrated in the laboratories concerned as specified in this paper.

\section{REFERENCES}

[1] Basma M. Mohammad El-Basioni, Sherine Mohamed Abd El-Kader and Hussein S. Eissa,"Independent Living for Persons with Disabilities and Elderly People Using Smart Home Technology", International Journal of Application or Innovation in Engineering \& Management (IJAIEM),vol 3, Issue 4, pp. 11-28, April 2014.

[2] Wei-Chung Teng, Yu-Chun Pao and Sheng-Luen Chung"Design of MyServer:a Residential Server in Smart Home Systems",2008 IEEE Asia-Pacific Services Computing Conference.

[3] Maarten Wijnants, Wim Lamote, Nicolas Letor, Chris Blondia, Eli De Poorter, Dries Naudts, Stijn Verstichel,
Bart Lannoo, Nelson Matthys and Christophe Huygens, "An Eco-friendly Hybrid Urban Computing Network Combining Community based Wireless LAN Access and Wireless Sensor Networking", 2012 IEEE International Conference on Green Computing and Communications, Conference on Internet of Things, and Conference on Cyber, Physical and Social Computing.

[4] HuanHui Yan andYusnita Rahayu, "Design and Development of Gas Leakage Monitoring System using Arduino and Zigbee", Proceedings of International Conference on Electrical Engineering,Computer Science and Informatics(EECSI 2014), Yogyakartha, Indonesia,20-21 August 2014.

[5] Kondamudi Siva Sai Ram, A.N.P.S Gupta, "IoT based Data Logger System for weather monitoring using Wireless sensor Networks", International Journal of Engineering Trends and Technology(IJETT) - vol 32,pp. 71-75, November 2- February 2016.

[6] Rajeev Piyare and Seong Ro Lee, "Towards Internet of Things(IoT): Integration of Wireless Sensor Network to Cloud Services for Data Collection and Sharing", International journal of Computer Networks \& Communications (IJCNC) vol.5, No.5,pp. 59-72, September 2013. 